\title{
Effects of Grinding Methods on Physicochemical Properties of Crude Polysaccharides from Phellinus baumii
}

\author{
Tingting $\mathrm{Li}^{1}$, Yan Yang${ }^{2}$, Linjun Chen ${ }^{1 *}$, Wanling Yang ${ }^{1 *}$ \\ ${ }^{1}$ Shanghai University of Medicine \& Health Sciences, Shanghai 2001318, China; \\ ${ }^{2}$ Shanghai Academy of Agricultural Sciences, Shanghai 201403, China
}

\begin{abstract}
The physicochemical properties of Phellinus baumii polysaccharides extracted from the fruiting bodies by different grinding methods were studied in this paper. Compared with ordinary grinding methods, superfine grinding technology exerts significantly higher yields of crude polysaccharides, polysaccharide contents and $\beta$-Glucan contents of six samples. HPSEC-MALLS- RI analysis shows the molecular weight distribution of P.baummii crude polysaccharides, which indicates superfine grinding technique generates higher molecular weight fractions and larger polydispersities. The 6 kinds of crude polysaccharides have obvious effects on NO generation of RAW264.7 cells, which indicated that crude polysaccharides from $P$. baumii may have some immune-enhancing effects. Meanwhile, CW30 and FP30 show the best immune enhancing effects. However, CW50 and CW70 exert weaker immune-enhancing effects compared with FP50 and FP70.
\end{abstract}

\section{INTRODUCTION}

Phellinus baumii Pilát belonging to Basidiomycota, Hymenomycetes, Aphyllophorales, Hymenochaetaceae and Phellinus, is a perennial, yellowish brown rare medicinal fungus[1]. The studies have shown that the Phellinus fruiting body has anti-tumor, immune-enhancing, hypoglycemic, antioxidant, anti-mutagenic, anti-mutation and other pharmacological effects [2-4]. Polysaccharide is one of the main active components of Phellinus. At present, the research on Phellinus polysaccharides mainly focuses on extraction technology, separation and purification, and its anticancer effect [5-7].

The superfine grinding technology is based on the principle of micron technology. The material is grinded, lashed, sheared, etc., and the material with $3 \mathrm{~mm}$ particle size or more is grinded to fine particles with 10 to $25 \mu \mathrm{m}$ particle diameters or less, thereby making the products have interface activity, and present special function. Powder with particle size below $25 \mu \mathrm{m}$ is usually called superfine powder, but traditional methods are difficult to grind solid materials to such fineness[8]. Numerous studies have shown that superfine grinding technology can improve the dissolution rate of effective components in medicines, increase the bioavailability of the medicine and save the medicine. Moreover, it is conducive to preserve bioactive components, improve the taste of traditional Chinese medicine (or food, health products) and so on [9]. In recent years, the research on superfine grinding technology is increasing with the development of new medicines, health product development, bioengineering, food processing, etc., which has been reported the superfine grinding is applied in the extraction of flavone in ginkgo leaves, Poria cocos polysaccharides, and functional properties of mushrooms, [10-12], but the effect of superfine grinding technology on the extraction of crude polysaccharides from Phellinus fruiting body and the physicochemical properties of polysaccharides has not been reported. This research selects the fruiting body of $P$.baumii to compare the effects of superfine grinding and ordinary grinding ways on the physicochemical properties of polysaccharide, and the bioactivites of polysaccharides extracted by grinded in two ways was compared, which provide basis for further research and utilization of Phellinus polysaccharide.

\section{MATERIALS AND METHODS}

\subsection{Source of materials}

Phellinus baumii Pilát 3249 strain was purchased from the Shanghai Edible Fungi Sub-center of the China Microorganisms Strain Collection, and the fruiting body were cultivated in Jinzhai County, Anhui Province.

* Corresponding author: Chenlj@sumhs.edu.cn; Yangwl@sumhs.edu.cn 


\subsection{Reagent}

Penicillin and streptomycin are products of Amersco Company, DMEM substratum, RPMI-1640, fetal bovine serum (FBS), pancreatin are products of Gibco Company, and Lipopolysaccharide (LPS) is product of Sigma Company; yeast $\beta$-dextran kit is the product of Ireland Megazyme Company; phenol, analytically pure distillation reagent; the rest reagents are domestic analytical pure reagents.

\subsection{Apparatus}

The main test apparatuses include the V-800 Rotary Evaporator of Büchi Vacuum Controller (BÜCHI Company), the freeze dryer (Thermo Savant Company), the Synergy HT multi-function enzyme mark instrument (Bio-Tek Company), HPLC(Waters Company), eight-angle laser light scatterometer and viscosity detectors (Wyatt Company), Dionex ICS 2500 high performance anion chromatograph (Dionex Company) and so on.

\subsection{Preparation of polysaccharide of fruiting body water-solubility}

Take two Phellinus fruiting body samples with $600 \mathrm{~g}$, one is grinded to wood bits shape by a traditional Chinese medicine grinder, and one part is carried out superfine grinding and passed through a 200-mesh screen. Add $6 \mathrm{~L}$ distilled water to the boiling water bath and extract $2 \mathrm{~h}$, the filter residue is extracted twice repeatedly and the extracts are combined and concentrated to $1 \mathrm{~L}$ by reduced pressure on a rotary evaporator, the concentrated solution is taken, $8000 \times \mathrm{g}$ is centrifuged for $30 \mathrm{~min}$ to remove the sediment, concentrated solution add absolute ethyl alcohol to ethyl alcohol with $30 \% 、 50 \% 、 70 \%$ concentration, place 12 h at $4{ }^{\circ} \mathrm{C}$, pour off the supernatant, the precipitated part is dissolved with $100 \mathrm{~mL}$ of distilled water. $8000 \times \mathrm{g}$ is centrifuged for $30 \mathrm{~min}$, remove insoluble matter, dialysis bag with $3500 \mathrm{Da}$ molecular interception is dialysis $2 \mathrm{~d}$, freeze-drying, and obtain water-soluble crude polysaccharide of Phellinus fruiting body.Crude polysaccharide of Phellinus fruiting body prepared by ordinary grinding, according to final concentration of precipitated ethyl alcohol, they are named FP30, FP50, and FP70, respectively. Crude polysaccharide prepared by superfine grinding fruiting body by same methods. They are named CW30, CW50, and CW70, respectively.

\subsection{Determination of crude polysaccharide yield and polysaccharide content}

The weight of crude polysaccharide after e balance weighed and freeze dried is analyzed; the crude polysaccharide yield is calculated [13].The total sugar content measured by the phenol sulfuric acid method [14].

\subsection{Determination of $\beta$-glucan content in water-soluble crude polysaccharides}

The crude polysaccharide after freeze-drying was weighed about $10 \mathrm{mg}$; consult the method of yeast and mushroom $\beta$-glucan detection kit provided by Megazyme, and carry put determination of $\beta$-glucan content

\section{7 molecular weight distribution of Phellinus polysaccharide is analyzed by HPSEC-MALLS-RI}

Weigh $2 \mathrm{mg}$ of the sample and dissolve it in $1 \mathrm{~mL}$ of mobile phase, the mobile phase is a solution with 0.05 $\mathrm{mol} / \mathrm{L} \mathrm{NaH} 2 \mathrm{PO}_{4}$ and $0.15 \mathrm{~mol} / \mathrm{L} \mathrm{NaNO}_{3}(\mathrm{pH}=7,0.02 \%$ sodium azide), and prepare a solution with a $2 \mathrm{mg} / \mathrm{mL}$ concentration. After $12000 \times \mathrm{g}$ is centrifuged for for 10 min, the supernatant is taken, after filtration through a $0.25 \mu \mathrm{m}$ aqueous microporous membrane, HPSEC-MALLS-RI analysis is carried out.

The analytical column selects TSK PWXL6000 and TSK PWXL3000 gel chromatography columns series to analyze, the flow speed is $0.5 \mathrm{ml} / \mathrm{min}$, the chromatography column temperature is constant at $35{ }^{\circ} \mathrm{C}$, and the wavelength of the laser detector light source selects $623.8 \mathrm{~nm}$. The refractive index increment $(\mathrm{dn} / \mathrm{dc})$ of the polysaccharide in solution is calculated according to $0.146 \mathrm{~mL} / \mathrm{g}$.

The light scattering data is collected and analyzed by Astra (version 6.1.1, Wyatt Technology, Santa Barbara, CA) data analysis software to calculate the molecular weight.

\subsection{Analysis of monosaccharide component of crude polysaccharide samples}

Implement by reference to literature [15] method.

\subsection{Effect of crude polysaccharide on the amount of NO released by RAW264.7 cells}

Weigh $5 \mathrm{mg}$ of crude polysaccharide component, respectively, add $1 \mathrm{~mL}$ PBS to prepare $5 \mathrm{mg} / \mathrm{mL}$ mother liquor, $15,000 \times \mathrm{g}$ is centrifuged for $30 \mathrm{~min}$, transfer the supernatant to another centrifuge tube, then take a certain amount and dilute to $200 \mu \mathrm{g} / \mathrm{mL}$ and $50 \mu \mathrm{g} / \mathrm{mL}$, and $10 \mu \mathrm{g} / \mathrm{mL}$ LPS is used as a positive control, PBS as a negative control, the production of NO released by macrophages is determined by reference[16].

\section{RESULTS AND ANALYSIS}




\subsection{Effect of grinding method on extraction yield of crude polysaccharide and content of $\beta$-glucan}

Compared with the ordinary grinding ways, the extraction yield and polysaccharide content of the Phellinus were significantly improved after superfine grinding (Table 1). The total yield of the crude polysaccharide obtained by ordinary grinding is only $0.97 \%$ (the sum of the yields of FP30, FP50 and FP70 in Table 1), and the total yield of the crude polysaccharide after superfine grinding is $4.48 \%$ (The sum of the yields of CW30, CW50 and CW70 in Table 1), compared with the ordinary grindimg ways, the yield of crude polysaccharide was increased by 3.6 times. For each precipitated component, the yield of CW30 is nearly 8 times higher than that of FP30, the yield of CW50 is 5 times that of FP50, and the yield of CW70 is twice that of FP70. The main component of the polysaccharide of the six components obtained by grading precipitation is $\beta$-glucan. The proportion of $\beta$-glucan in the crude polysaccharide extracted after fruiting body is carried out superfine pulverization, its proportion increases as the concentration of alcohol precipitate increases, while the proportion of $\beta$-glucan in the crude polysaccharide extracted after ordinary grinding decreases as the concentration of alcohol precipitation decreases. It shows that there are significant differences for crude polysaccharide components obtained by different grinding ways.

Table.1 Effect of different grinding methods on yields, polysaccharide content and $\beta$-glucan content of P.baummii crude ploysaccharides

\begin{tabular}{cccc}
\hline $\begin{array}{c}\text { Crude poly- } \\
\text { saccharides }\end{array}$ & $\begin{array}{c}\text { Crude } \\
\text { polysaccharide } \\
\text { s yields }(\%)\end{array}$ & $\begin{array}{c}\text { Polysaccharide } \\
\text { content }(\%)\end{array}$ & $\begin{array}{c}\beta \text {-Glucan content } \\
(\%)\end{array}$ \\
\hline CW30 & 1.18 & $71.02 \pm 0.73$ & $51.54 \pm 0.68$ \\
CW50 & 2.23 & $66.43 \pm 1.25$ & $58.37 \pm 0.53$ \\
CW70 & 1.07 & $85.54 \pm 0.45$ & $78.55 \pm 1.06$ \\
FP30 & 0.14 & $45.20 \pm 0.46$ & $41.59 \pm 1.23$ \\
FP50 & 0.43 & $43.04 \pm 0.58$ & $31.11 \pm 0.86$ \\
FP70 & 0.40 & $50.60 \pm 1.05$ & $20.91 \pm 0.59$ \\
\hline
\end{tabular}

\subsection{Effect of grinding ways on molecular weight distribution of crude polysaccharide of Phellinus}

The HPSEC-MALLS-RI analysis diagram of polysaccharide component of Phellinus fruiting body is shown in Figure 1. The distribution range of molecular weight of each polysaccharide component obtained by the superfine grinding method is wide, and distribution range of component molecular weight of the ordinary grinding is narrow. As shown in Figure.1, both FP30 and CW30 mainly contain three peaks, and the peak time is similar, and CW30 is slightly earlier. The three peaks of obtained component CW30 after superfine grinding, and the molecular weights were similar, so that it was difficult to obtain effective separation, and the three peaks of FP30 had a narrow molecular weight distribution range and molecular weights are significantly different. This may be because superfine grinding causes some effects on the structure of the polysaccharide and make some glycosidic bonds of the polysaccharide to be broken, thereby forming many similar components of molecular weight. Moreover, as shown in Figure. 2 and Figure.3, the peak time of the CW50 and CW70 two components is in advance relative to the polysaccharide component of the ordinary grinding ways, this may be that superfine grinding contributes to the dissolution of macromolecular polysaccharides.

The molecular weight of each component of the crude polysaccharide of Phellinus was calculated by Astra data analysis software, the results are shown in Table 2. The results showed that CW30 had more sugar peaks and concentrated distribution, through the analysis of the three main peaks of CW30, it showed that the weight average molecular weight of Peak1 was $2.201 \times 10^{6} \mathrm{Da}$, which was slightly larger than the $1.895 \times 10^{6}$ Da of Peak1 of FP30. Moreover, the molecular weight of Peak2 and Peak3 of CW30 is larger than that of FP30 (Fig.1a). Fig.1b shows that the CW50 and FP50 samples have only one main peak, and the weight average molecular weights are $3.614 \times 10^{5} \mathrm{Da}$ and $3.089 \times 10^{4} \mathrm{Da}$, respectively, the molecular weight of CW50 is much larger than FP50, in addition, the polydispersity index of CW50 is 2.463 , which is much larger than 1.306 of FP50, it shows that CW50 has a broad molecular weight distribution, which may be composed of many polysaccharides with similar molecular weights, while the component of FP50 is relatively simple. The liquid phase data for the CW70 and FP70 samples showed the same trend (Fig 1.c).

Comparison of the two treatment methods found that superfine grinding contributes to the dissolution of macromolecular polysaccharides; moreover, it destroys the structure of macromolecular polysaccharides, cause some glycosidic bond to break, and form many similar components of molecular weight. Therefore, the crude polysaccharide component obtained by superfine grinding is more complicated in composition than the ordinary grinding, and it is difficult to achieve the separation and purification effect. 

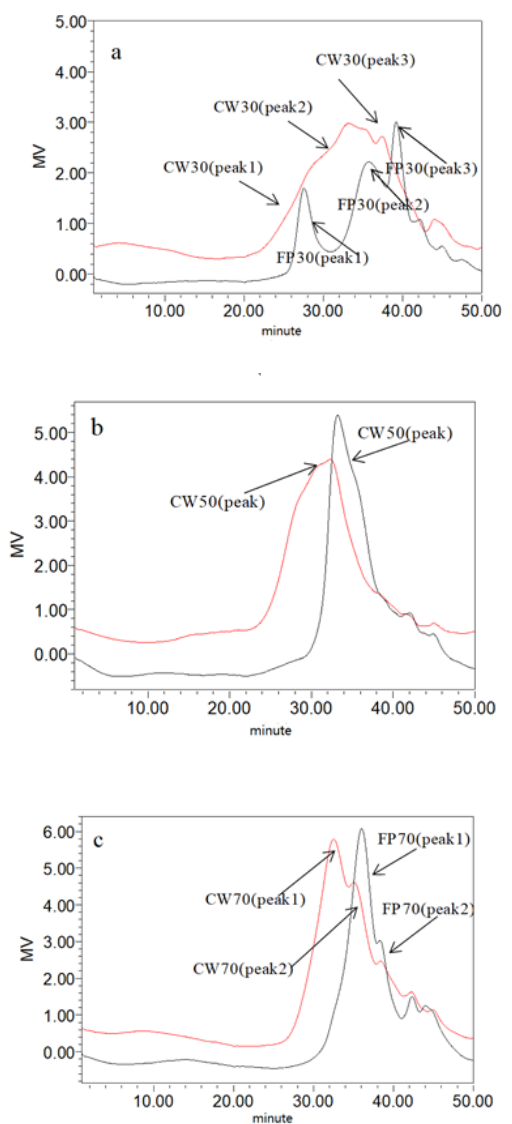

Fig. 1 HPLC chromatograms of crude polysaccharides extracted from P.baummii fruiting bodies ground by different methods a .HPLC chromatogram of CW30 and FP30; b. HPLC chromatogram of CW50 and FP50; c .

HPLC chromatogram of CW70 and FP70

Table 2 Molecular weight distributions of crude polysaccharides extracted from P.baummii fruiting bodies ground by different methods

\begin{tabular}{|c|c|c|c|}
\hline & $\begin{array}{l}\text { Peak } \\
\text { number }\end{array}$ & $\begin{array}{c}\text { weight-average } \\
\text { molecular } \\
\text { weightMw } \\
\text { (Da) }\end{array}$ & $\begin{array}{c}\text { number } \\
\text { average } \\
\text { molecular } \\
\text { weightMn } \\
\text { (Da) } \\
\end{array}$ \\
\hline CW30 & $\begin{array}{l}\text { Peak1 } \\
\text { Peak2 } \\
\text { Peak3 }\end{array}$ & $\begin{array}{l}2.201 \times 10^{6} \\
1.387 \times 10^{5} \\
4.456 \times 10^{4}\end{array}$ & $\begin{array}{l}1.635 \times 10^{6} \\
1.197 \times 10^{5} \\
4.345 \times 10^{4}\end{array}$ \\
\hline FP30 & $\begin{array}{l}\text { Peak1 } \\
\text { Peak2 } \\
\text { Peak3 }\end{array}$ & $\begin{array}{l}1.895 \times 10^{6} \\
1.293 \times 10^{5} \\
2.518 \times 10^{4}\end{array}$ & $\begin{array}{l}1.685 \times 10^{6} \\
8.348 \times 10^{4} \\
2.195 \times 10^{4}\end{array}$ \\
\hline CW50 & Peak & $3.614 \times 10^{5}$ & $1.468 \times 10^{5}$ \\
\hline FP50 & Peak & $3.089 \times 10^{4}$ & $2.364 \times 10^{4}$ \\
\hline CW70 & $\begin{array}{l}\text { Peak1 } \\
\text { Peak2 }\end{array}$ & $\begin{array}{l}7.771 \times 10^{4} \\
3.547 \times 10^{4}\end{array}$ & $\begin{array}{l}6.105 \times 10^{4} \\
3.219 \times 10^{4}\end{array}$ \\
\hline FP70 & $\begin{array}{l}\text { Peak1 } \\
\text { Peak2 } \\
\end{array}$ & $\begin{array}{l}4.033 \times 10^{4} \\
1.989 \times 10^{4} \\
\end{array}$ & $\begin{array}{l}2.430 \times 10^{4} \\
1.425 \times 10^{4} \\
\end{array}$ \\
\hline
\end{tabular}

\subsection{Analysis of monosaccharide composition of crude polysaccharides of Phellinus fruiting body}

Six components of polysaccharides from Phellinus fruiting body extracts after grading precipitation obtained by different grinding way, the molar percentage results of monosaccharide composition obtained after high performance anion exchange chromatography (HPAEC) detection is shown in Table 3. As can be seen from Table 3, the monosaccharide compositions of the six polysaccharide components are basically the same, they all contain fucose, galactose, glucose and mannose four monosaccharides, however, the proportion of each monosaccharide in each component is different, glucose has the highest proportion, the next are galactose and mannose, proportion of fucose is the lowest. Moreover, the proportion of glucose in each component obtained after superfine grinding is increased compared with the component of ordinary grinding, which is consistent with the higher content of the superfine grinding component $\beta$-glucan in Table 1 . It may be that superfine grinding causes the cell wall to break and a large amount of $\beta$-glucan on the cell wall dissolve out.

Table 3 Molar ratio of monosaccharide of six samples

\begin{tabular}{lllll}
\hline Fractions & $\begin{array}{l}\text { Fucose } \\
(\%)\end{array}$ & $\begin{array}{l}\text { Galactose } \\
(\%)\end{array}$ & $\begin{array}{l}\text { Glucose } \\
(\%)\end{array}$ & $\begin{array}{l}\text { Mannose } \\
(\%)\end{array}$ \\
\hline CW30 & 0.84 & 2.09 & 92.17 & 4.90 \\
CW50 & 1.79 & 6.43 & 79.58 & 12.20 \\
CW70 & 1.69 & 4.85 & 83.26 & 10.20 \\
FP30 & 1.61 & 5.77 & 86.11 & 6.51 \\
FP50 & 8.02 & 22.32 & 52.86 & 16.80 \\
FP70 & 5.37 & 15.77 & 50.44 & 28.42 \\
\hline
\end{tabular}

\subsection{Effect of crude polysaccharide samples on the amount of NO released by RAW264.7 cell}

The results of in vitro activity test of crude polysaccharides of Phellinus fruiting body by different grinding methods are shown in Fig.2. The results showed that the six Phellinus polysaccharide components obtained by the two grinding ways all stimulate macrophages to release $\mathrm{NO}$ in vitro. CW30 and FP30 are comparable in activity and both show good concentration dependence. The activities of CW50 and CW70 are significantly lower than those of FP50 and FP70, which may be related to the type and structure of polysaccharides, and further research is needed. 


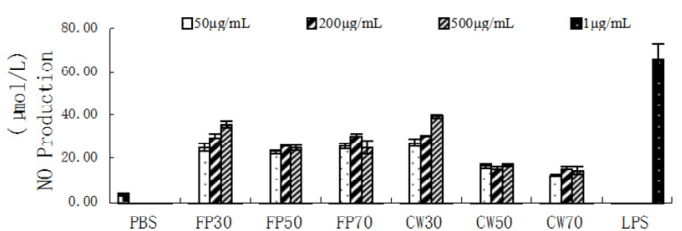

Fig.2 Effects of crude polysaccharides extracted from P.baummii fruiting bodies ground by different methods and step ethanol precipitation on NO release from RAW264.7 cells

\section{DISCUSSION}

At present, there are many products such as glossy ganoderma superfine powder and Phellinus superfine powder on the market, but the physicochemical property and biological activity of the main component polysaccharide of this superfine powder have not been reported. This paper takes Phellinus super fine powder as the research object, compares the differences of physicochemical property and bioactivity of Phellinus fruiting body extracted from different grinding ways, it was found that the crude polysaccharide of Phellinus obtained after superfine grinding treatment, there is a significant increase in polysaccharide yield, polysaccharide content and $\beta$-glucan content. Therefore, the superfine grinding technology is applied to the pretreatment of Phellinus polysaccharide extraction, which can greatly increase the yield of Phellinus polysaccharide.

In recent years, studies have shown that $\beta$-glucan has immunoregulatory function and anti-tumor activities, which is an important polysaccharide in edible fungi, the edible fungus $\beta$-glucan is mainly derived from edible, medicinal burden fungi and cell wall of ascus saccharomycetes. In the superfine treatment process, cell wall polysaccharides (mainly $\beta$-glucan) are dissolved out [17], which may be an important reason for the high content of $\beta$-glucan in the superfine powder of Phellinus. The fruiting body polysaccharides before and after superfine grinding have significant differences in monosaccharide composition molar ratio and molecular weight distribution; compared with the conventional grinding wasys, the vitro activity of the Phellinus polysaccharide obtained by superfine grinding was slightly decreased, it may be that the superfine grinding process has a certain destructive effect on the structure of its polysaccharide, thereby affecting its bioactivity. Experimental studies have found that the crude polysaccharide component obtained by superfine grinding has a broad molecular weight distribution, and it is difficult to achieve the separation and purification effect. Therefore, the superfine grinding technology is suitable for industrial large-scale preparation of Phellinus polysaccharide pretreatment, thereby increasing the yield of polysaccharides and reducing the cost of Phellinus polysaccharide; it is not suitable for the research on separation and purification of polysaccharides and structural analysis of related homogeneous polysaccharides.

\section{Acknowledgement}

Foundation items: "Chenguang Program" supported by Shanghai Education Development Foundation and Shanghai Municipal Education Commission (14CGB21) ; The Young Teachers Foundation of Shanghai Municipal Education Committee; The "Seed Fund" Foundation of Shanghai University of Medicine \& Health Sciences (HMSF-17-22-008 、 SFP-18-22-05-003); The "Teaching Project" of Shanghai University of Medicine \& Health Sciences (JC(18) 05-C2-01);

\section{References}

1. Huang N L, Lin Z B, Chen GL, et al. Medicinal and Edible Fungi. Shanghai: Scientific and Technical Documentation Press, 2010: 1690-1693.

2. Zhang L F, Zou L. The research progress of polysaccharides of Phellinus. Edib Fun Chin, 2012, 31(4):1-4.

3. Yang Y, Ye L B, Zhang J S. Structural analysis of a bioactive polysaccharide, PISP1, from the medicinal mushroom Phellinus igniarius. Biosci Biotechnol Biochem. 2009: 73(1):134 - 139.

4. CAO C L. Biological characteristics and polysaccharide structure of Phellinus mori. Beijing: Beijing Forestry University, PhD. 2012

5. HSIEH P W, WU J B, WU Y C. Chemistry and biology of Phellinus linteus. BioMedicine. 2013: 3(3):106-113.

6. LIU A J, CHEN W W, WANG W H, et al. Optimization extraction of polysaccharid es from Phellinus linteus. Food Research and Developent. 2006, 27(10): 32-35.

7. HE J C, HAN Y P. Ultrafiltration membrane separation technology and its application in polysaccharide extraction and purification. Edul Fun, 2005, (1): 5-8.

8. ZHANG J, YU Y, XU G H. Technology of superfine grinding and its application in food industry.Journal of Agri Sci 2010, 31(1):51-54.

9. YANG L W, ZHAO X Y, LI T, et al. Ultrafine Grinding and Its Effects on Traditional Chinese Medicine. World science technology/modernization of traditional Chinese medicine and material medica. 2008, 10(6):77-81.

10. HUANG Q C, LIN M X, HONG Y P, et al. Effect of Ultrafine Grinding on the Dissolution of Total Flavonoids in Ginkgo biloba Leaves. Journal of Anhui Agri Sci. 2012, 40(10): 5884-5885. 
11. MEI G M, LI F J, SHEN S(), et al. Extraction of Alkali Soluble Polysaccharide in Poriacocos and Study on Ultramicro Smashing Modification. Food Scien. 2007, 28(10):278-282.

12. GAO H, SHI D F, HE J J, et al. Effect of Superfine Grinding on Functional Components and Properties of Lentinus edodes Stems. Food Scien. 2010, 31(5):40-43.

13. JIANG J. Study on polysaccharides extraction、 decoloration technology and biological activity from the fruiting body of Hericium Erinaceous. Nanjing: Nanjing Agriculture University, MSc. 2012.

14. ZHANG W J. Sugar complex biochemical research technology. Zhejiang: Zhejiang University Press, 2003.

15. Yang Y, Zhang J S, Liu Y F, et al. Structural elucidation of a 3-O-methyl-galactose-containing neutral polysaccharide from fruiting bodies of Phellinus igniarius. Carbohydrate research, 2007, 342, 1063-1070.

16. KIM G Y, CHOI G S, LEE S H, PARK Y M, et al. Acidic polysaccharide isolated from Phellinus linteus enhances through the up-regulation of nitric oxide and tumor necrosis factor-alpha from peritoneal macrophages. J Ethnopharmacol. 2004, 95: 69-76.

17. SUN P L, YANG K, ZHANG AQ. Reserch advancement of $\beta$-glucan in edible medical mushroom. Edib Fun China, 2008. 27(1):9-13 